\title{
DETERMINAÇÃO DO TURNO DE REGA PARA A PRODUÇÃO DE MUDAS DE PITAIA EM AMBIENTE PROTEGIDO
}

\author{
Antonio Robson Moreira ${ }^{*}$, Fernanda Ludmyla Barbosa de Souza ${ }^{2}$, Raimundo Thiago Lima da Silva ${ }^{3}$, Raimundo Leonardo Lima de \\ Oliveira $^{4}$, Airton dos Santos Alonço ${ }^{1}$, Leane Castro de Souza ${ }^{5}$, Dauto Pivetta Carpes ${ }^{1}$ \\ ${ }^{1}$ Departamento de Engenharia Rural, Universidade Federal de Santa Maria, 97105-900, Santa Maria, Brasil. \\ ${ }^{2}$ Departamento de Agronomia, Universidade Estadual do Oeste do Paraná, 85960-000, Marechal Cândido Rondon, Brasil. \\ ${ }^{3}$ Departamento de Agronomia, Universidade Federal Rural da Amazônia, 68650-000, Capitão Poço, Brasil. \\ ${ }^{4}$ Departamento de Solos e Adubos, Universidade Estadual Paulista, 14884-900, Jaboticabal, Brasil. \\ ${ }_{5}^{5}$ Departamento de Agronomia, Universidade Federal Rural da Amazônia, 66077-830, Belém, Brasil.
}

*E-mail: $\underline{\text { robson.n.13@hotmail.com }}$

\section{RESUMO}

Este trabalho teve como objetivo avaliar a influência do turno de rega sobre o desenvolvimento de mudas de pitaia, produzidas em ambiente protegido, assim como estabelecer um intervalo de tempo em dias, confiável entre as irrigações. O experimento foi realizado na área experimental da Universidade Federal Rural da Amazônia, Campus Capitão Poço, Pará. O delineamento experimental utilizado foi o inteiramente casualizado (DIC), com sete tratamentos constituídos pelos seguintes turnos de rega: T1 - sem irrigação durante todo o período de avaliação; T2 - turno de rega de um dia; T3 - turno de rega de dois dias; T4 - turno de rega de três dias; T5 - turno de rega de quatro dias; T6 - turno de rega de cinco dias; T7 - turno de rega de seis dias; com doze repetições, totalizando oitenta e quatro unidades experimentais. Aos vinte e sete dias, após a implantação do experimento, com a emissão dos primeiros cladódios pelas mudas, foi iniciada a mensuração dos parâmetros biométricos: altura, número de cladódios emitidos, comprimento e diâmetro do cladódio de primeira ordem, e comprimento do cladódio de segunda ordem. A irrigação das mudas proporcionou incremento significativo nos valores de altura, número de cladódios emitidos e no comprimento e diâmetro dos cladódios de primeira ordem, maioria dos parâmetros biométricos avaliados, em comparação com a testemunha. O turno de rega de três dias (T4) foi o tratamento que garantiu melhor uso consultivo da água, apresentando os pontos de máxima para altura, comprimento e diâmetro do cladódio de primeira ordem.

Palavras-chave: Engenharia de água e solo, Cactaceae, Metabolismo CAM.

\section{Introdução}

Pertencente à família Cactaceae, a pitaia é uma espécie frutífera originária da América Central e cultivada em maior escala no Oriente Médio e Ásia. Apresenta como principais atrativos aos agricultores sua alta produtividade de até 34 toneladas por hectare $\left(\mathrm{t} \mathrm{ha}^{-1}\right)$ [1], com perspectiva de obter produção logo no primeiro ano de implantação do pomar [2-3] e preço de comercialização alto no mercado de frutas exóticas.

É destaque entre as frutíferas consideradas de cultivo promissor, e que em meados da década passada, passou a suprir uma demanda significativa do mercado de frutas exóticas no continente Europeu [3]. A pitaia vem sendo procurada não só pelo exotismo da aparência, mas, principalmente, por suas características organolépticas [4-5], tais como: sabor doce e suave, polpa firme e sementes com ação laxante.
A principal característica de adaptação morfológica desse grupo de plantas é o caule denominado cladódio, típico de plantas xerófilas que tem como funções realizar fotossíntese, efetuar trocas gasosas e armazenar água. As plantas dessa família são capazes de tolerar calor e frio intenso, além de longos períodos de estiagem e solos de baixa fertilidade natural. Apresentam, em sua estrutura, modificações no caule para armazenamento de água, ausência de folhas, superfícies recobertas com ceras naturais e abertura noturna dos estômatos para a absorção de dióxido de carbono (metabolismo $\mathrm{CAM}^{1}$ ), permitindo que as mesmas tolerem condições adversas de clima [6].

Apesar de a pitaia pertencer a um grupo de plantas bastante tolerantes a períodos longos de estiagem, submetê-la a diferentes cenários de disponibilidade hídrica podem trazer informações relevantes em relação com qual frequência é

\footnotetext{
${ }^{1}$ Metabolismo Ácido das Crassuláceas
} 
necessária fornecer água (turno de rega) e o quanto tolerante ela também poder ser ao excesso hídrico.

É preciso considerar, também, que o correto manejo de sistemas de irrigação deve proporcionar condições adequadas, no que diz respeito à disponibilidade de água, com o objetivo de potencializar o desenvolvimento e, consequentemente a produtividade das culturas, além de maximizar a eficiência de uso da água, evitando desperdício e reduzindo os custos de investimento e operação, de tal forma que a atividade se torne economicamente viável e sustentável do ponto de vista ambiental [7].

A irrigação bem como a grande maioria das tecnologias aplicadas a produção agrícola, pode proporcionar modificações e impactos sobre o ambiente. Conhecer detalhadamente a aplicação dessa técnica permite quantificar os impactos causados, assim como visualizar possíveis soluções para otimizar o uso de um recurso ambiental tão importante como água.

Ao longo dos últimos anos, parte dos estudos relacionados à produção de mudas de pitaia, têm sido concentrados, em sua grande maioria, em estabelecer ou pelo menos sugerir, um intervalo confiável para o comprimentos de partes vegetativas, utilizadas para propagação [3,5,8-9] e sua influência sobre o desenvolvimento inicial das mesmas, sendo escassos ou inexistentes os estudos de investigação, quanto ao seu comportamento em relação à disponibilidade hídrica e consequente influência sobre os parâmetros biométricos de crescimento.

Diante do exposto, o trabalho teve como objetivo avaliar a influência do turno de rega sobre o desenvolvimento de mudas de pitaia, produzidas em ambiente protegido, assim como estabelecer um intervalo de tempo em dias, confiável entre as irrigações.

\section{Metodologia}

\subsection{Localização da área do estudo}

$\mathrm{O}$ experimento foi realizado na área experimental da Universidade Federal Rural da Amazônia, Campus Capitão Poço, Pará (UFRA-CCP), com coordenadas geográficas de latitude $01^{\circ} 44^{\prime} 47^{\prime}$ ' $\mathrm{S}$ e longitude $47^{\circ} 03^{\prime} 34^{\prime \prime} \mathrm{W}$, em estufa plástica, com dimensões $16 \mathrm{~m} \times 30 \mathrm{~m}$.

\subsection{Material e métodos}

As estacas utilizadas como materiais propagativos foram provenientes de plantas matrizes, cultivadas no pomar da Universidade Federal Rural da Amazônia, campus Capitão Poço Pará (UFRA-CCP). Todas as estacas possuíam 0,3 metro (m) de comprimento e foram cultivadas em vasos plásticos próprios para mudas com volume 5 litros (1), preenchidos com substrato a base de terra preta, esterco bovino e casca de arroz carbonizado na proporção de 1:1:1. Para mensurar os parâmetros biométricos, foram utilizados fita métrica e paquímetro digital de precisão 0,01 milímetro (mm).

O delineamento experimental utilizado foi o inteiramente casualizado (DIC), com sete tratamentos, constituídos pelos seguintes turnos de regas: T1 - sem irrigação durante todo o período de avaliação; T2 - turno de rega de um dia; T3 - turno de rega de dois dias; T4 - turno de rega de três dias; T5 - turno de rega de quatro dias; T6 - turno de rega de cinco dias; T7 - turno de rega de seis dias, com doze repetições, totalizando oitenta e quatro unidades experimentais.

A irrigação das mudas foi realizada manualmente e de forma indiferente nos primeiros quatro dias iniciais e, após esse período foram aplicados os tratamentos. Aos vinte e sete dias após a implantação do experimento com a emissão dos primeiros cladódios pelas mudas, foi iniciada a mensuração dos parâmetros biométricos: altura (AL) (maior distância vertical entre a superfície do solo e a extremidade superior do cladódio mais alto), número de cladódios emitidos (NC), comprimento do cladódio de primeira ordem (CC1), diâmetro do cladódio de primeira ordem e comprimento do cladódio de segunda ordem (DC1 e CC2), respectivamente.

\subsection{Análises dos dados}

Os dados foram submetidos aos testes de KolmogorovSmirnov e Bartlett $(\mathrm{P}<0,05)$ para testar a aderência dos resíduos à distribuição normal e identificar a condição de homogeneidade das variâncias. O conjunto de dados que, previamente, não atenderam a qualquer um dos pressupostos, foram transformados de acordo com a expressão $\left(\mathrm{Y}_{\mathrm{ij}}=\sqrt{ } \mathrm{Y}_{\mathrm{ij}}\right)$. Constatadas as condições de normalidade e homogeneidade, procedeu-se a aplicação do teste de Dunnett e a análise de regressão $(\mathrm{P}<0,05)$, nos Softwares Action Stat $^{\circledR}$ versão 3.2.60.1118 build 1 e Sisvar $^{\circledR}$ versão 5.3 beta [10], respectivamente. Gráficos com modelos significativos foram gerados no Software Microsoft Excel ${ }^{\circledR}$.

\section{Resultados e discussões}

\subsection{Comparação entre testemunha e turnos de rega}

As variáveis altura das mudas (AL), número de cladódios emitidos (NC), comprimento do cladódio de primeira ordem e diâmetro do cladódio de primeira ordem (CC1 e DC1), respectivamente, sofreram influência dos turnos de rega, apresentando diferença estatística em comparação à testemunha pelo teste de Dunnett, tabela 1.

\subsection{Resultados da análise de regressão}

O número de cladódios emitidos (NC) e o comprimento do cladódio de segunda ordem (CC2) não apresentaram modelos matemáticos que se ajustassem ao comportamento apresentado $(\operatorname{Pr}>0,05)$. No entanto, os parâmetros altura (AL), comprimento e também o diâmetro do cladódio de primeira ordem $(\mathrm{CC} 1$ e 
DC1), respectivamente, apresentaram comportamento descritos pelo modelo polinomial, de segundo grau $(\operatorname{Pr} \leq 0,01)$, tabela 2 .

O ponto de máxima para a altura das mudas, bem como de comprimento e diâmetro do cladódio de primeira ordem, foi verificado no turno de rega de três dias (T4), conforme derivada primeira dos modelos polinomiais de segundo grau (Figuras 1, 2 e 3). Estes resultados confirmam os obtidos por Queiroz et al. [11], que não encontraram diferença para altura, largura e número de cladódios, em estudo realizado com palma forrageira, sob diferentes lâminas de irrigação, conduzido na região do semiárido do estado de Pernambuco. Porém, verificaram tendência de decréscimo nos valores absolutos dessas variáveis, conforme houve o acréscimo na disponibilidade hídrica.
Tabela 1. Resultados para aplicação do teste de Dunnett.

\begin{tabular}{cccccc}
\hline $\begin{array}{c}\text { Diferença } \\
\text { entre níveis }\end{array}$ & $\begin{array}{c}\text { AL }(\mathrm{cm}) \\
\text { Pr }>\text { F }\end{array}$ & $\begin{array}{c}\text { NB } \\
\text { Pr F }\end{array}$ & $\begin{array}{c}\text { CC1 }(\mathrm{cm}) \\
\text { Pr }>F\end{array}$ & $\begin{array}{c}\text { DC1 }(\mathrm{cm}) \\
\text { Pr }>F\end{array}$ & $\begin{array}{c}\text { CC2 }(\mathrm{cm}) \\
\text { Pr }>F\end{array}$ \\
\hline T2 - T1 & $0,001^{* *}$ & $0,484^{\text {ns }}$ & $0,000^{* *}$ & $0,000^{* *}$ & $0,184^{\text {ns }}$ \\
T3 - T1 & $0,002^{* *}$ & $0,013^{*}$ & $0,001^{* *}$ & $0,001^{* *}$ & $0,063^{\text {ns }}$ \\
T4 - T1 & $0,000^{* *}$ & $0,287^{\text {ns }}$ & $0,000^{* *}$ & $0,001^{* *}$ & $0,325^{\text {ns }}$ \\
T5 - T1 & $0,000^{* *}$ & $0,720^{\text {ns }}$ & $0,000^{* *}$ & $0,000^{* *}$ & $0,904^{\text {ns }}$ \\
T6 - T1 & $0,091^{\text {ns }}$ & $0,378^{\text {ns }}$ & $0,025^{*}$ & $0,023^{*}$ & $0,958^{\text {ns }}$ \\
T7 - T1 & $0,349^{\text {ns }}$ & $0,484^{\text {ns }}$ & $0,064^{\text {ns }}$ & $0,037^{*}$ & $0,124^{\text {ns }}$ \\
\hline
\end{tabular}

${ }^{* *}$ Significativo a $1 \%$ de probabilidade de erro $(\operatorname{Pr} \leq 0,01)$

${ }^{*}$ Significativo a $5 \%$ de probabilidade de erro $(\operatorname{Pr} \leq 0,05)$

${ }^{\text {ns }}$ Não significativo $(\operatorname{Pr}>0,05)$

Tabela 2. Resultados da análise de regressão para os parâmetros Altura (AL), Número de brotos (NB), Comprimento do cladódio de primeira ordem (CC1), Diâmetro do cladódio de primeira ordem (DC1) e Comprimento do cladódio de segunda ordem (CC2).

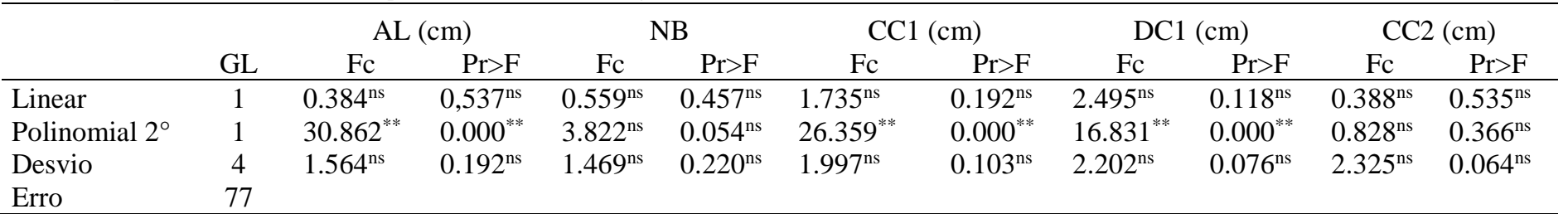

** Significativo a $1 \%$ de probabilidade de erro $(\operatorname{Pr} \leq 0,01)$

* Significativo a $5 \%$ de probabilidade de erro $(\operatorname{Pr} \leq 0,05)$

${ }^{\text {ns }}$ Não significativo $(\operatorname{Pr}>0,05)$

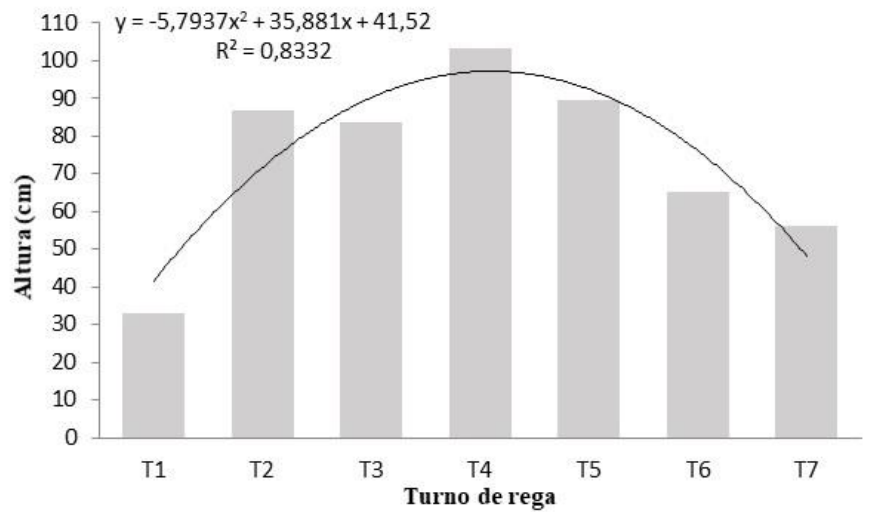

Figura 1: Modelo polinomial de $2^{\circ}$ para a altura (AL), em função dos turnos de rega utilizados para a produção de mudas sob ambiente protegido.

Redução da capacidade de uso da água para o crescimento da planta, neste caso, pode ser atribuída ao fato de as espécies pertencentes à família Cactaceae apresentarem baixo consumo de água, contribuindo dessa forma, para a manutenção de elevado conteúdo de água no solo [12], e desta maneira inibindo seu desenvolvimento, principalmente quando submetidas a turnos de rega com menor intervalo de tempo entre uma irrigação e outra. No presente trabalho, a maior disponibilidade hídrica proporcionada por turnos de regas menores, além de inibir o desenvolvimento das mudas de pitaia, causaram podridão de alguns cladódios.

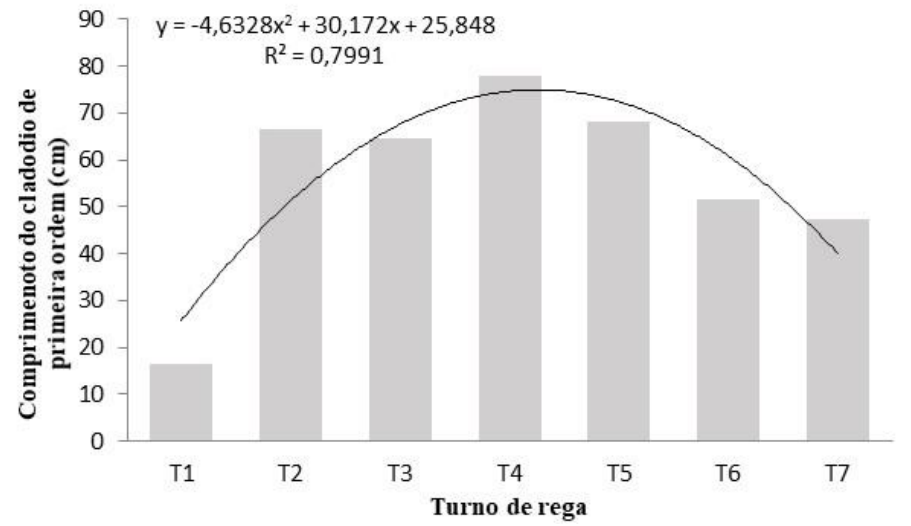

Figura 2: Modelo polinomial de $2^{\circ}$ para o comprimento do cladódio de primeira ordem (CC1), em função dos turnos de rega utilizados para a produção de mudas sob ambiente protegido.

De maneira geral, a maioria dos turnos de rega adotados, apresentaram resultados positivos sobre as variáveis biométricas de desenvolvimento das mudas, quando comparados à testemunha, pois apesar de a pitaia ser uma espécie com baixa demanda hídrica, ela apresenta necessidade hídrica permanente, assim como o 
abacaxi, outra frutífera com metabolismo CAM, que responde positivamente à irrigação [13-14].

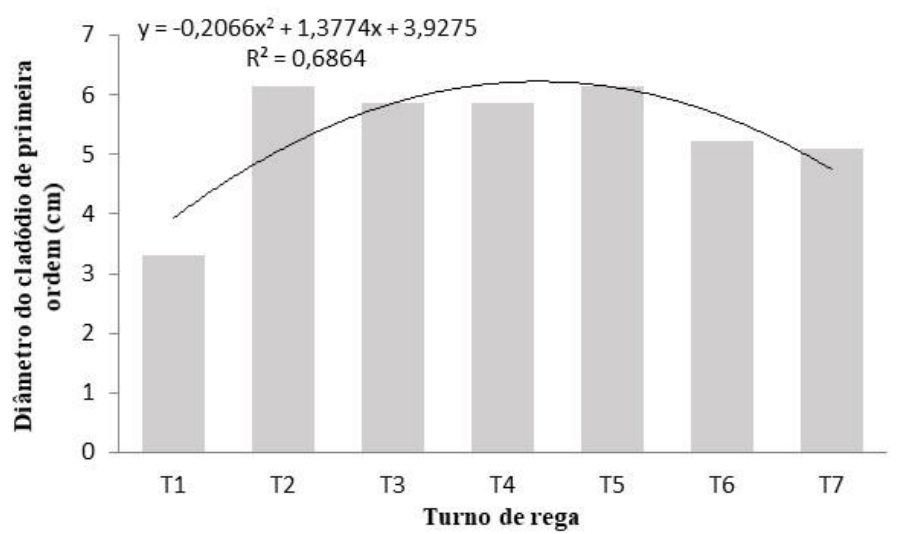

Figura 3: Modelo polinomial de $2^{\circ}$ para o diâmetro do cladódio de primeira ordem (DC1), em função dos turnos de rega utilizados para a produção de mudas sob ambiente protegido.

Com base nos resultados obtidos é possível afirmar que a adoção do turno de rega de três dias (T4), foi aquele que proporcionou os melhores resultados no desenvolvimento inicial das mudas de pitaia, quando comparado aos demais tratamentos.

\section{Conclusões}

A irrigação das mudas produzidas em ambiente protegido, proporcionou incremento nos valores absolutos de altura, número de cladódios emitidos, comprimento e diâmetro dos cladódios de primeira ordem, em comparação à testemunha.

O turno de rega de três dias (T4), foi o tratamento que garantiu melhor desempenho no uso consultivo da água, apresentando os pontos de máxima para altura, comprimento e diâmetro do cladódio de primeira ordem.

\section{Agradecimentos}

À Universidade Federal Rural da Amazônia - UFRA.

\section{DETERMINATION OF IRRIGATION SCHEDULING FOR THE PRODUCTION OF PITAHAYA SEEDLINGS IN A PROTECTED ENVIRONMENT}

ABSTRACT: This work aimed to assess the influence of the irrigation scheduling on the development of pitahaya seedlings, produced in a protected environment, as well as to establish a reliable time interval in days between the irrigations. The experiment was conducted in the experimental area of the Amazon Rural Federal University, Campus Capitão Poço, Pará. The experimental design was completely randomized (CRD), with seven treatments consisting of the following irrigation scheduling options: T1 - without irrigation during the whole period of evaluation; T2 - one-day irrigation schedule; T3 - two-day irrigation schedule; T4 - three-day irrigation schedule; T5 - fourday irrigation schedule; T6 - five-day irrigation schedule; T7 six-day irrigation schedule, with twelve replicates, totaling eightyfour experimental units. Twenty-seven days after the implantation of the experiment, with the emission of the first cladodes by the seedlings, the biometric parameters were measured: height, number of cladodes emitted, length and diameter of first order cladode, and length of second order cladode. The irrigation of the seedlings provided a significant increase in the values of height, number of cladodes emitted and in the length and diameter of the first order cladodes, most of the biometric parameters assessed, in comparison with the control. Three-day irrigation schedule (T4) was the treatment that provided the best consultative use of water, presenting maximum scores for height, length and diameter of the first-order cladode.

Keywords: Water and soil engineering, Cactaceae, Crassulacean Acid Metabolism.

\section{Referências}

[1] NERD, A.; TEL-ZUR, N.; MIZRAHI, Y. Fruit of vine and columnar cacti. In: NOBEL, P. S. (Ed.). Cacti: biology and uses. Los Angeles: UCLA, p. 254-262, 2002 .

[2] ZEE, F.; YEN, CHUNG-RUEN; NISHINA, M. Pitaya (Dragon fruit, Straw berry pearl). Fruits e Nuts, Hawai, n. 9, pag. 1-3, 2004.

[3] LE BELLEC, F.; VILLANT, F.; IMBERT, E. Pitahaya (Hylocereus spp.): a new crop, a market with a future. Fruits, France, Vol. 61, n. 4, p. 237 -250. 2006.

[4] SILVA, Marco T. H.; MARTINS, Antonio B. G.; ANDRADE, Renata A. Enraizamento de estacas de pitaya vermelha em diferentes substratos. Revista Caatinga, Vol.19, n.1, p.61-64, 2006.

[5] MARQUES, V. B. et al. Fenologia reprodutiva de pitaia vermelha no município de Lavras, MG. Ciência Rural, Vol.41, n.6, p.984-987, 2011.

[6] MARENCO, R. A.; LOPES, N. F. Fisiologia vegetal: fotossíntese, respiração, relações hídricas e nutrição mineral. 3 ed. (atual. ampl.). Viçosa: UFV, 2009. 486 $\mathrm{p}$

[7] CANTUÁRIO, Fernando Santos. Produção de pimentão submetido a estresse hídrico e silicato de potássio em cultivo protegido. 2012. 93 f. Dissertação (Mestrado em Agronomia) - Universidade Federal de Uberlândia, 2012.

[8] BRAGA, M. F. et al. Enraizamento de estacas de três espécies silvestres de Passiflora. Revista Brasileira de Fruticultura, Jaboticabal, Vol. 28, n. 2, p. 284-288, 2006

[9] MOREIRA, A, R. et al. Determinação do comprimento da estaca para a produção de mudas de pitaia (Hylocereus costaricensis) em ambiente protegido. Tecno-Lógica, Vol. 21, n. 2, p. 41-45, 2017.

[10] FERREIRA, D. F. SISVAR - Sistema de análise de variância. Versão 5.3. Lavras-MG: UFLA, 2010. 
[11] QUEIROZ, M. G. de. et al. Características morfofisiológicas e produtividade da palma forrageira em diferentes lâminas de irrigação. Revista Brasileira de Engenharia Agrícola e Ambiental, Vol.19, n.10, p.931-938, 2015.

[12] SILVA, T. G. F. et al. Indicadores de eficiência do uso da água e de nutrientes de clones de palma forrageira em condições de sequeiro no Semiárido brasileiro. Revista Bragantia, Vol. 73, p. 184-191, 2014.

[13] ALMEIDA, O.A. de, et.al. Influência da irrigação no ciclo do abacaxizeiro cv. Pérola em área de Tabuleiro Costeiro. Revista Brasileira de Fruticultura, Vol. 24, n. 2, p. 431-435, 2002.

[14] MELO, A. S. de. et al. Desenvolvimento vegetativo, rendimento da fruta e otimização do abacaxizeiro cv. Pérola em diferentes níveis de irrigação. Ciência Rural, Vol. 36, n. 1, p. 93-98, 2006. 